\title{
Photonic crystal nanolaser monolithically integrated with passive waveguide for effective light extraction
}

\author{
Kengo Nozaki, ${ }^{\text {a) }}$ Hideki Watanabe, and Toshihiko Baba ${ }^{\text {b) }}$ \\ Department of Electrical and Computer Engineering, Yokohama National University, 79-5 Tokiwadai, \\ Hodogayaku, Yokohama 240-8501, Japan and Core Research for Evolutional Science and Technology \\ (CREST), Japan Science and Technology (JST) Agency, 5, Sanbancho, Chiyodaku, Tokyo 102-0075, \\ Japan
}

(Received 14 November 2007; accepted 14 December 2007; published online 14 January 2008)

\begin{abstract}
We recently reported room-temperature continuous-wave operation in a GaInAsP photonic crystal slab nanolaser. In this letter, we demonstrate effective light extraction from the nanolaser monolithically integrated with a passive waveguide by using a GaInAsP butt-joint regrowth technique. Theoretically, the extraction efficiency through the waveguide was calculated to be $80 \%$ for the optimum design of the coupling system of the nanolaser and the waveguide. In the experiment, we evaluated a differential quantum efficiency of $4 \%$, which was degraded mainly due to the detection loss of the output light. (C) 2008 American Institute of Physics.
\end{abstract}

[DOI: $10.1063 / 1.2831916$ ]

Photonic crystal (PC) point defect nanolasers ${ }^{1-3}$ with ultrasmall modal volumes $V_{m}$ are anticipated to be used as local light sources in high-density photonic integrated circuits. We recently fabricated a point-shift PC nanolaser consisting of a shift of only two lattice points in a PC slab containing a GaInAsP quantum well active layer (called the $H 0$ nanolaser), and we succeeded in demonstrating roomtemperature (RT) continuous-wave (cw) operation. ${ }^{4}$ The fitting of theoretical results to experimental values indicated that the laser mode has one primary antinode at the cavity center, with $V_{m}=0.019 \mu \mathrm{m}^{3}$, which is close to the smallest limit for optical cavities. ${ }^{5}$ However, although it has never been quantitatively evaluated, people expect the output power and the efficiency of such nanolasers to be far below practical levels because of their small size and because their output light is strongly diffracted. Other groups have described candidate device configurations for light extraction, such as the coupling of the nanolaser with a PC line defect waveguide, ${ }^{6,7}$ vertical beaming of the laser light by structural tuning, ${ }^{8}$ and evanescent coupling of the laser mode with a tapered optical fiber. ${ }^{9,10}$ The latter two are effective for light extraction to external systems. However, integration with the PC waveguide is more advantageous in the case of potential photonic integrated circuits based on a PC slab. It should be noted that when the waveguide is formed in the active region, efficient light extraction and low loss optical wiring are not expected due to the strong interband absorption. To avoid this, we developed an active-passive-integrated PC slab, wherein a GaInAsP quantum well slab was connected with a wide bandgap quaternary bulk slab by using a butt-joint metal-organic chemical vapor deposition (MOCVD) regrowth technique. We previously demonstrated the coupling system of a line defect laser and passive line defect waveguide, and evaluated a differential quantum efficiency of $8 \%$ in laser operation. ${ }^{11}$ In this letter, we demonstrate the monolithic integration of the $H 0$ nanolaser and passive line defect

\footnotetext{
${ }^{a)}$ Electronic mail: d05sd106@ynu.ac.jp.

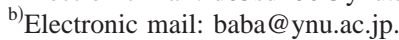

waveguide, as well as the effective extraction of laser light from the waveguide.

First, we calculated the light coupling between the nanolaser and the waveguide using the three-dimensional finitedifference time-domain (FDTD) method. Figure 1(a) shows the schematic of the $H 0$ nanocavity and the line defect waveguide in a triangular lattice PC slab. We assumed a slab index $n$ of 3.4, a lattice constant $a$ of $480 \mathrm{~nm}$, a normalized airhole diameter $2 r / a$ of 0.54 , a normalized slab thickness $d / a$ of 0.42 , and a normalized airhole shift $s / a$ of 0.17 . Under these conditions, the $H 0$ nanocavity maintains the mono-

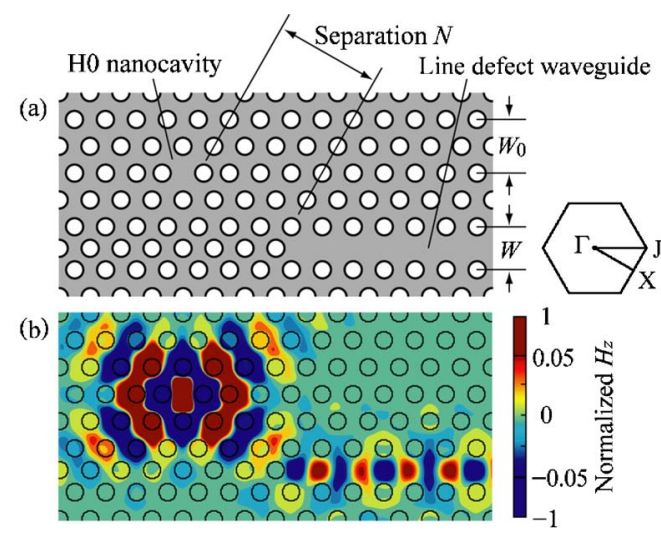

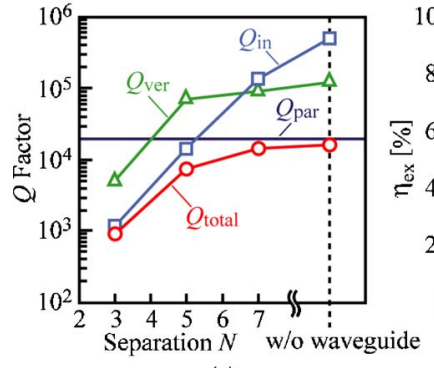

(c)

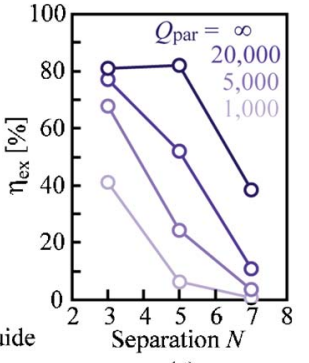

(d)
FIG. 1. (Color online) FDTD calculation results for the coupling system of the $H 0$ nanocavity and the PC line defect waveguide. (a) Top view of the device model. (b) Field $\left(H_{z}\right)$ distribution. (c) Dependence of cavity $Q$ factors on separation $N$. (d) Dependence of light extraction efficiency $\eta_{\text {ex }}$ on separation $N$. 
pole mode with $V_{m}=0.019 \mu \mathrm{m}^{3}=0.15(\lambda / n)^{3}$, where $\lambda$ is the modal wavelength in vacuum. In the coupling system, the line defect waveguide is placed toward the direction that the monopole mode strongly penetrates. ${ }^{12}$ If the waveguide width $W$ was same as the width $W_{0}$ of the simple line defect waveguide, the lasing mode takes several coupling points with an even and odd waveguide modes in the leaky region. Therefore, $W$ was moderately reduced to obtain a unique coupling condition with the even mode in the guiding region. In order to balance the high cavity $Q$ and extraction efficiency, the separation $N$ denoting the number of airholes between the cavity and the waveguide in $\Gamma-X$ direction was also changed. Figure 1(b) shows the modal field distribution in the coupling system for $N=5$ and $W=0.8 W_{0}$ when the nanocavity was excited at the monopole mode frequency. The cavity mode is clearly coupled to the waveguide. Figure 1(c) shows the calculation results for the $Q$ factor with separation $N$. Here, $Q_{\mathrm{ver}}, Q_{\mathrm{in}}$, and $Q_{\mathrm{par}}$ are determined, respectively, by the out-of-plane radiation loss in the vertical direction, in-plane coupling to the waveguide, and parasitic losses caused by the free carrier absorption and the scattering loss due to the imperfection of the device. We set $Q_{\mathrm{par}}=20000$, which was experimentally evaluated under the RT cw operation condition. ${ }^{4}$ As shown in Fig. 1(c), $Q_{\text {in }}$ exponentially decreases as $N$ decreases. $Q_{\text {ver }}$ also decreased when $N$ was less than five, because the out-of-plane radiation loss is sensitive to the field profile penetrating from the cavity to the PC. However, the total cavity $Q$, given as $Q_{\text {total }}=\left(Q_{\mathrm{ver}}^{-1}+Q_{\text {in }}^{-1}\right.$ $\left.+Q_{\mathrm{par}}^{-1}\right)^{-1}$, is mainly limited by $Q_{\text {in }}$ when $N<5$. Figure $1(\mathrm{~d})$ shows the light extraction efficiency from the nanocavity, $\eta_{\mathrm{ex}}=Q_{\text {total }} / Q_{\mathrm{in}}$, where $Q_{\mathrm{par}}$ is an unknown variable. $\eta_{\mathrm{ex}}$ increased with decreasing $N$ and increasing $Q_{\text {par }}$ because the parasitic losses were reduced relatively. For $N=3$ and $Q_{\text {par }}$ $=20000, \eta_{\mathrm{ex}}$ is expected to be $80 \%$ with $Q_{\text {total }}$ being maintained to be nearly $10^{3}$.

In the experiment, we prepared a GaInAsP activepassive-integrated wafer formed by the MOCVD butt-joint regrowth process, the details of which are described in Ref. 11. The slab layer consisted of an active region with six compressively strained quantum wells with a photoluminescence peak at $\lambda=1.555 \mu \mathrm{m}$ and a $1.32 \mu \mathrm{m}$ quaternary bulk passive region. The active/passive butt-joint boundary of the PC slab was also formed with a negligible position error in the vertical direction and a small projection with a height $<10 \mathrm{~nm}$, which was formed at the initial stage of the regrowth process. PC airholes were formed by e-beam lithography and $\mathrm{HI} / \mathrm{Xe}$-inductively coupled plasma etching, ${ }^{13}$ and the airbridge structure was formed by selective $\mathrm{HCl}$ wet etching. The nanolaser and the waveguide were clearly formed with a smooth etching profile, as shown in Fig. 2(a). In the measurement, the nanolaser was photopumped at RT by $0.98 \mu \mathrm{m}$ wavelength pulsed laser light with a duty ratio of $0.075 \%$ and a focused spot diameter of $2.5 \mu \mathrm{m}$. The light emitted from the facet of the waveguide was directly detected by an as-cleaved multimode fiber GI-50 and observed using an optical spectral analyzer. Figures 2(b) and 2(c) show the laser characteristics for a sample with $N=3$ and $W=0.8 W_{0}$. The effective pump power $P_{\text {eff }}$ was evaluated from the absorption efficiency of the pump light in the slab (we estimated it to be 30\%) (Ref. 14) and the overlap efficiency of the pump spot with the cavity area (we estimated this to be $10 \%$ when the cavity area is assumed to be an ellipse attaching the innermost airholes). The maximum Downloaded 22 Jan 2008 to 216.1.176.121. Redistribution subject

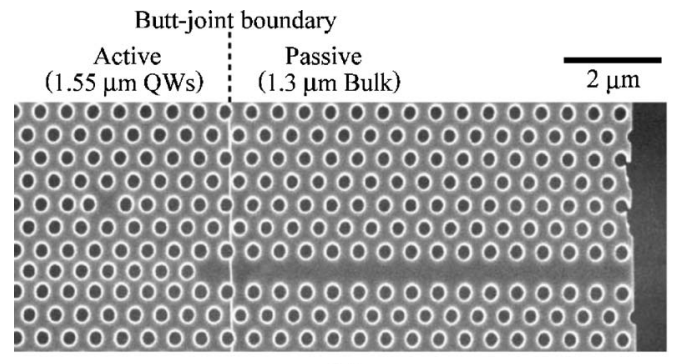

(a)

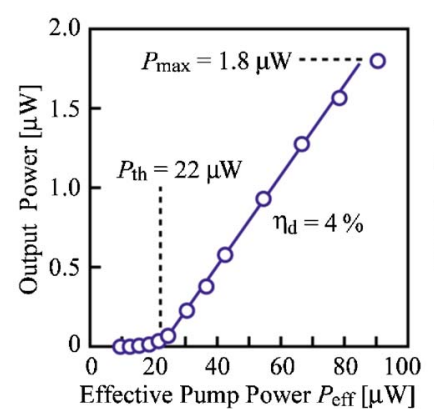

(b)

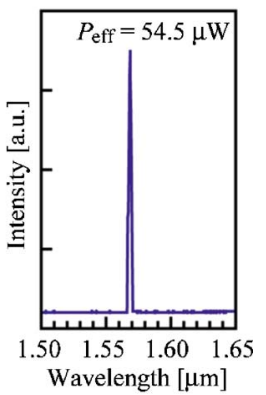

(c)
FIG. 2. (Color online) Experimental results. (a) Top view of the fabricated device. (b) Output power vs effective pump power characteristic. (c) Lasing spectrum above the threshold.

output power $P_{\max }$, threshold pump power $P_{\mathrm{th}}$, and differential quantum efficiency $\eta_{d}$ were estimated to be $1.8 \mu \mathrm{W}$, $22 \mu \mathrm{W}$, and $4 \%$, respectively. $\eta_{d}$ is not a real value of the device but dominantly includes the coupling loss between the waveguide and the detection fiber. If such a coupling loss is reduced by using a lensed fiber, this value will be improved to $\sim 30 \%$. Although such measurement was not realized in this study because of the degradation of the device, more than a sevenfold improvement in $\eta_{d}$ has been confirmed for PC line defect lasers. ${ }^{15}$ However, this is still 2.7 times lower than the calculated light extraction efficiency $\eta_{\mathrm{ex}}$. This difference cannot be due to the propagation loss in the waveguide since the waveguide length was shorter than $10 \mu \mathrm{m}$. One reason for this must be an internal quantum efficiency lower than $100 \%$. Other reasons considered include a lower $\eta_{\mathrm{ex}}$ of the fabricated device and the reflection and/or wide-angle diffraction of light at the waveguide facet. A small fabrication error in the structural parameters affects the depth and orientation of the field penetration from the cavity to the waveguide, resulting in lower $\eta_{\mathrm{ex}}$. Also, the reflection and diffraction are sensitive to the position of the cleaved facet of the waveguide with respect to the airhole pattern of the $\mathrm{PC},{ }^{16}$ which could further degrade the detection efficiency.

Figure 3(a) compares the laser characteristics for some samples with different $N$ ranging from 3 to 7 . Obviously, the maximum output power and $\eta_{d}$ increase as $N$ decreases. Figure 3(b) summarizes the dependence of $P_{\text {th }}$ and $\eta_{d}$ on $N . P_{\text {th }}$ also increases as $N$ decreases because of the decrease in $Q_{\text {in }}$; $P_{\text {th }}$ is $6 \mu \mathrm{W}$ without the waveguide and $\sim 20 \mu \mathrm{W}$ for $N=3$. The behavior of $\eta_{d}$ is very similar to that of $\eta_{\mathrm{ex}}$ in Fig. 1(d) for $Q_{\mathrm{par}}=1000-5000$. Some fluctuations in the experimental plots observed even for the same $N$ might be due to fabrication error, as mentioned above. By reducing the fabrication error and optimizing the waveguide facet, further improvement and stable evaluation of $\eta_{d}$ can be expected. A $\eta_{d}$ of $80 \%$ with a low threshold of a few tens of microwatts is to AIP license or copyright; see http://apl.aip.org/apl/copyright.jsp 


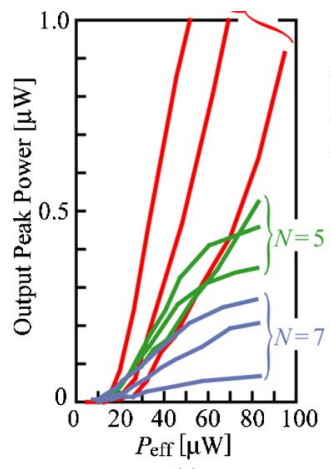

(a)

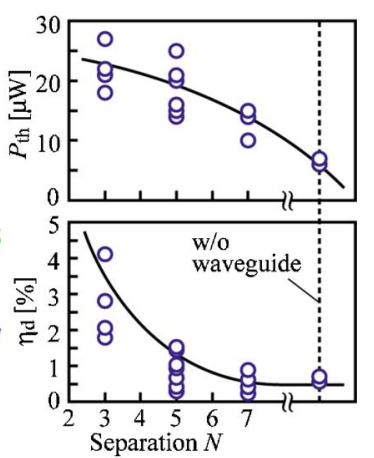

(b)
FIG. 3. (Color online) Dependence of lasing characteristics on separation $N$. (a) Lasing characteristics observed for samples with different $N$. (b) Threshold $P_{\text {th }}$ and differential quantum efficiency $\eta_{d}$.

theoretically obtainable for $N=3$ and $Q \sim 10^{3}$. In the case of using an active material such as a single quantum well or a quantum dot having a lower modal gain, a higher $Q_{\text {total }}$ of $10^{4}$ order for $N \geqslant 5$ is preferable to obtain a lower threshold of microwatt order while maintaining $\eta_{d}>50 \%$.

In conclusion, we demonstrated an ultrasmall $H 0$ nanolaser integrated with a passive waveguide in an activepassive-integrated GaInAsP slab for effective light extraction. Theoretical calculations showed that the light extraction efficiency from the laser to the waveguide can be $80 \%$ by careful design of the coupling system. Experimentally, we fabricated the device and observed RT lasing operation by photopumping. The differential quantum efficiency of the laser light was evaluated to be $4 \%$. However, the effective value without detection loss was considered to be $\sim 30 \%$, and this value can be further improved by the optimization of the structure and the fabrication process. This is a promising result for future dense photonic integration of nanolasers, waveguides, and other functional photonic devices.

This work was supported by a Grant-in-Aid and a Research Fellowship of the Japan Society for the Promotion of Science.

${ }^{1}$ O. Painter, R. K. Lee, A. Scherer, A. Yariv, J. D. O’brien, P. D. Dapkus, and I. Kim, Science 284, 1819 (1999).

${ }^{2}$ H. Y. Ryu, S. H. Kim, H. G. Park, J. K. Hwang, Y. H. Lee, and J. S. Kim, Appl. Phys. Lett. 80, 3883 (2002).

${ }^{3}$ T. Baba, D. Sano, K. Nozaki, K. Inoshita, Y. Kuroki, and F. Koyama, Appl. Phys. Lett. 85, 3989 (2004).

${ }^{4}$ K. Nozaki, S. Kita, and T. Baba, Opt. Express 15, 7506 (2007).

${ }^{5}$ K. Nozaki, and T. Baba, Appl. Phys. Lett. 88, 211101 (2006).

${ }^{6}$ W. Zheng, X. Ma, G. Ren, X. Cai, L. Chen, K. Nozaki, and T. Baba, Quantum Electronics and Laser Science Conference, Long Beach, 2006 (unpublished), Paper No. QTuL6.

${ }^{7}$ A. Faraon, E. Waks, D. Englund, I. Fushman, and J. Vučković, Appl. Phys. Lett. 90, 073102 (2007).

${ }^{8}$ S. H. Kim, S. K. Kim, and Y. H. Lee, Phys. Rev. B 73, 235117 (2006).

${ }^{9}$ K. Srinivasan, P. E. Barclay, M. Borselli, and O. Painter, Phys. Rev. B 70, 081306 (2004).

${ }^{10}$ I. K. Hwang, S. K. Kim, J. K. Yang, S. H. Kim, S. H. Lee, and Y. H. Lee, Appl. Phys. Lett. 87, 131107 (2005).

${ }^{11} \mathrm{H}$. Watanabe, and T. Baba, Electron. Lett. 42, 695 (2006).

${ }^{12}$ G. H. Kim, Y. H. Lee, A. Shinya, and M. Notomi, Opt. Express 12, 6624 (2004).

${ }^{13}$ T. Ide, J. Hashimoto, K. Nozaki, E. Mizuta, and T. Baba, Jpn. J. Appl. Phys., Part 2 45, L102 (2006).

${ }^{14}$ K. Nozaki, A. Nakagawa, D. Sano, and T. Baba, IEEE J. Sel. Top. Quantum Electron. 9, 1355 (2003).

${ }^{15} \mathrm{H}$. Watanabe, K. Nozaki, and T. Baba, IEEE Lasers Electro-Optics Society Annual Meeting, Orlando (2007), No. MN3.

${ }^{16}$ P. Kramper, M. Agio, C. M. Soukoulis, A. Birner, F. Müller, R. B. Wehrspohn, U. Gösele, and V. Sandoghdar, Phys. Rev. Lett. 92, 113903 (2004). 